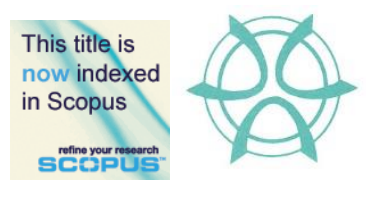

PLANNING MALAYSIA:

Journal of the Malaysian Institute of Planners

VOLUME 15 ISSUE 2 (2017), Page 13 - 24

\title{
THE FINANCIAL COSTS OF URBAN SPRAWL: CASE STUDY OF PENANG STATE
}

\author{
Shahriza Osman ${ }^{1,2}$, Jamalunlaili Abdullah $^{3}$, \& Abdul Hadi Nawawi ${ }^{4}$ \\ ${ }^{1,3,4}$ Faculty of Architecture, Planning \& Surveying \\ UNIVERSITI TEKNOLOGI MARA (UiTM) \\ ${ }^{2}$ College of Business \\ UNIVERSITI UTARA MALAYSIA
}

\begin{abstract}
The financial cost of urban sprawl is the additional or incremental costs measured relative to the type, density and location of sprawl development compared to inner city development. The costs are incurred by both the public and private sectors. Numerous studies on costs of sprawl found that there is an increase in infrastructure costs associated with sprawl development compared to compact development. Sprawl increases infrastructure costs in several ways. Lower density means each yard of linear infrastructure such as water and sewer serves fewer households. Housing type and location affect the number of water and sewer laterals and resultant costs. Road network cost increases as well. The increase in costs compels researchers to examine what type and which location of development should be encouraged. This paper adopts a case study approach in examining housing development costs of eleven housing projects in Penang State, Malaysia. Mathematical and statistical analysis are applied on actual data. The results of cross tabulation reveal that costs per unit of housing development, based on traditional development calculations, are cheaper with greater distance from CBD. However, when additional development costs data (infrastructure costs such as roadworks, sewerage and water lines from housing projects to the sub-service centres) are factored in, the results show that the cost per unit is higher with greater distance from CBD. These results support international findings that cost per unit of development rises as distance increases and densities decreases, characteristics of sprawl development. This is perhaps the first empirical results on financial costs of sprawl in Malaysia and hope to be a springboard to future studies on costs of urban sprawl in Malaysia.
\end{abstract}

Keywords: financial costs, urban sprawl, housing development, Penang State

Date Received: $26^{\text {th }}$ July 2016

Date of Acceptance: $6^{\text {th }}$ July 2017

${ }^{1}$ Senior Lecturer at Universiti Utara Malaysia. Email: shahriza@uum.edu.my 
Shahriza Osman, Jamalunlaili Abdullah, \& Abdul Hadi Nawawi

The Financial Costs of Urban Sprawl: Case Study of Penang State

\section{INTRODUCTION}

Sprawl is an urban phenomenon characterised by low density outward extension into undeveloped areas. Leapfrog development compels the construction of two sets of infrastructure that are underused (Burchell, 1990). Cost of sprawl studies claim that significant cost savings regarding infrastructure supply could be realized if a better planned and more compact urban development is achieved (Thomson, Hoffman \& Staniforth, 2003). Furthermore, Bryant and Eves' (2014) research findings support the proposition that developers' payment of infrastructure charges are passed to homebuyers. These are significant contributors to reduced housing affordability. Besides that, a growing literature on costs argue that sprawl is not economically efficient because it creates a host of private, public and social costs that are not adequately captured through market processes (Frank, 1989).

In Malaysia, urban growth is expected to intensify and Malaysia will not return to its former status of a rural country, due to the rapid growth of the nations' economy and the theory of elasticity of demand (Abdullah, 2009). Several studies in Malaysia have shown the existence of urban sprawl in major metropolitan areas in Malaysia (Abdullah, 2003; Yaakup \& Sulaiman, 2005; Yahaya, 2006; Safudin, 2007). These studies used a number of accepted indicators which have been used worldwide to quantify urban sprawl such as comparison of population growth rate between main urban settlement and town periphery areas, and comparative analysis of built-up areas and population growth. Spatial evidences have been carried out by Sabri and Yaakup (2003), Noor and Rosni (2010), and Noor, Asmawi and Rusni (2014). The urban areas are expanding rapidly and there are evidences of the existence of the phenomenon of urban sprawl in the three largest Metropolitan areas of Malaysia which are Kuala Lumpur, Penang and Johor Bharu. The urban sprawl phenomenon in Malaysia is associated with low density development in greenfield areas (Abdullah et al, 2009).

Research of urban sprawl in Malaysia tends to focus on physical characteristics, and no studies have been carried out on costs of urban sprawl in the country. To spearhead research on urban sprawl cost in Malaysia, this paper examines the cost of housing development projects in various locations in Penang State.

\section{RESEARCH BACKGROUND}

Many researchers have found that there were substantial costs incurred by allowing sprawl development (Isard \& Coughlin, 1957; Frank 1989; RERC 1989; Burchell \& Shad, 1998; Speir \& Stephenson, 2002). It is costly especially in terms of providing public infrastructure and services such as roads and sewer. The early study by Isard and Coughlin (1957) found that cost per lot for sewer lines and roads increases with average lot size. Colorado Natural Resources (2000) used mathematical model to measure costs and the results showed that unit costs are 
PLANNING MALAYSIA

Journal of the Malaysia Institute of Planners (2017)

higher with lower densities. Stanley (2006) used the General Linear model to calculate costs and the results revealed that land cost increased with lot size, water lines and roads. Provision of public services is more expensive for urban sprawl compared to other patterns of development (Burchell \& Shad, 1998; Ladd, 1998; Duncan, 1992; Ewing, Pendall \& Chen, 2002; Ojima, 2007).

A growing literature on costs of urban sprawl argues that sprawl is not economically efficient because it creates a host of private, public, and social costs that are not adequately captured through market forces (Frank, 1989). Researchers discovered that considerable cost for savings can be materialized by enhancing urban population densities and locating new development near existing well-built areas. Burchell and Shad (2003) show that infrastructure costs associated with sprawl is higher than the costs of conventional suburban development. For roads, compact development costs 75 percent of the cost for conventional development. One issue related to sprawl is the corresponding increase in costs for infrastructures which are usually borne by the government.

Urban sprawl phenomenon is destroying the urban landscape environmentally, socially, economically and politically. The social, economic and environmental costs have been studied widely in the West but financial costs received limited research coverage. In Malaysia there is lack of study to quantify the cost of sprawl especially in providing public services and residential development. The need to evaluate the financial cost of sprawl is even more pressing in Malaysia since the country has the fourth-largest built-up land in East Asia as of 2010. The country's urban land grew from about 3,900 to 4,600 square kilometres between 2000 and 2010, an average annual growth rate of $1.5 \%$. Its urban population increased during this period from 10.2 million ( $43 \%$ of the total population) to 15 million (53\%), making it among the more urbanized countries and economies in the region, after Japan, the Republic of Korea, Singapore, and Taiwan.

\section{METHODOLOGY}

The research method is exploratory in nature, using mixed research design. In the first stage, it was important to understand sprawl development of the study area. For this, data were obtained through secondary sources such as population data records from the Statistic Department of Malaysia and other related government publications. Data on financial costs were collected from primary and secondary sources. The researchers start with property reports to select suitable housing projects, and then case study was used in order to examine actual urban sprawl phenomenon and related financial costs.

Eleven housing projects were identified from five regions in Penang. Urban sprawl was identified by calculating and comparing the percentage change in population growth rate to the percentage change in built-up area. The quantification of the financial costs were determined by estimating additional 
Shahriza Osman, Jamalunlaili Abdullah, \& Abdul Hadi Nawawi

The Financial Costs of Urban Sprawl: Case Study of Penang State

costs to the housing development projects, which included land cost and infrastructure costs (roads, sewerage, water and electricity), among others. Descriptive statistics such as frequency distribution, Cronbach's Alpha, mean and standard deviation were used to analyse the data collected. Cross tabulation analysis was also carried out.

\section{LIMITATIONS OF STUDY}

This research relies on data gathered from professionals and local authorities involved in the projects due to reluctance of some developers to provide data. Some of the calculations on costs are based on assumptions derived from similar developments. It is also assumed that infrastructure extensions are carried from the nearest CBD, namely Georgetown and Butterworth.

\section{FINDINGS AND RESULTS}

Due to rapid urbanization in Penang State since the late 1980s, the districts in Penang (Seberang Perai Tengah and Seberang Perai Selatan on the mainland, and Barat Daya on the island) have experienced sprawl development. Urban sprawl in Penang is due mainly to low density development in mostly greenfield areas.

Higher sprawl regions in Penang are found to be significantly correlated with higher population growth and land consumption growth rate. This may be due to higher consumer's preference for residential in the mainland as house prices on the island are high. House price in the city centre is even higher; one of the reasons is the land price in city centre is very expensive and this affect housing development in city centre. Apartments are more affordable by residents who seek housing at an affordable price.

Figure 1 shows the locations of eleven housing projects included in this study. These included ten single storey housing projects and an apartment building of 480 units. Three locations on Penang Island have high costs per unit of housing and are in high density areas. One of the reasons for the high costs per unit of housing is the land costs in city centre are higher compared to the mainland. 
PLANNING MALAYSIA

Journal of the Malaysia Institute of Planners (2017)

Figure 1 Locations of Actual Development Project Cost per Unit in Penang

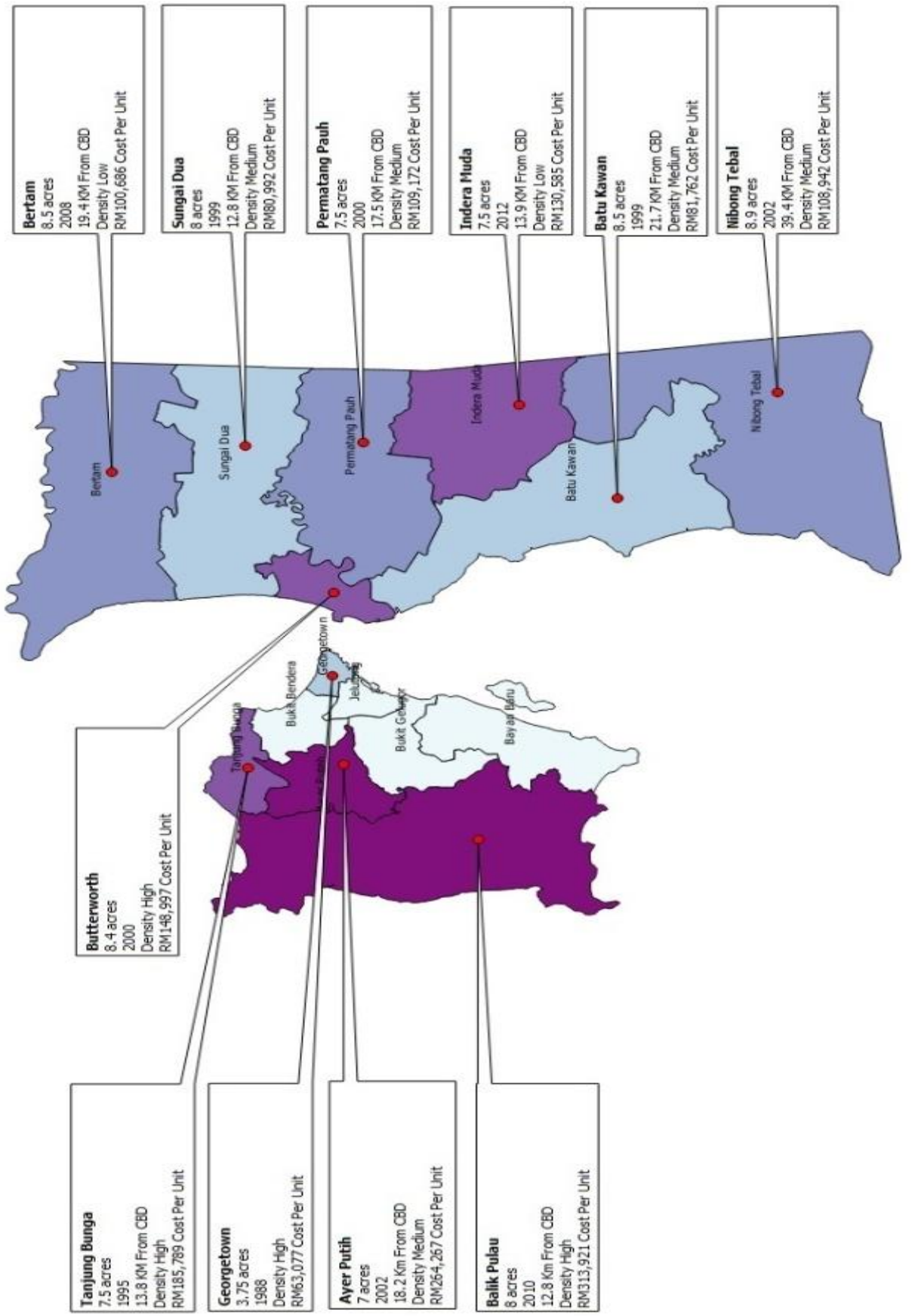


Shahriza Osman, Jamalunlaili Abdullah, \& Abdul Hadi Nawawi

The Financial Costs of Urban Sprawl: Case Study of Penang State

The net areas for the housing development projects were between 3.75 acres to 8.9 acres. The years of completion for each project were from 1988 till 2012. The number of housing units ranged from 90 units to 150 units for single storey terrace houses and one 480 units of apartment block in George Town. Distance from Central Business District (CBD) was calculated from Butterworth in the mainland and Georgetown in Penang Island. The mean distance for all 11 projects from the closest CBD was 14.5 kilometres.

This research calculates actual development costs incurred by the developers of these housing projects as shown in Table 1 . These costs included "normal" variable costs such as the land, buildings, infrastructure within the project sites, professional fees, contribution to the local authority and contingencies fee.

The land costs were based on the market value in that particular locations, which range from RM469,750 to RM7,400,000. On site infrastructure costs varied between RM1,238,677 to RM8,779,000. Professional fees ranged from RM 279,800 to RM1,241,600. Contribution to the local authority ranged from RM125,000 to RM387,300. These variables were considered as normal costs of the developments. The total costs of these normal variables ranged from RM $8,909,220$ to RM31,755,250 for the eleven housing projects. Dividing the total costs to the number of housing unit yielded the cost per unit of houses to range from RM80,992 to RM313,92.

Figure 2 shows the result of cross tabulation of all eleven projects based on their distances from the CBDs and cost per unit. The mean cost per unit was RM142,753. Houses above the mean was considered as high cost, while those below the mean were considered as low cost. With mean distance of 14.5 kilometres, it was found that 6 housing projects were considered as short distance (below the mean) and the other 5 housing projects as long distance (above the mean). 
PLANNING MALAYSIA

Journal of the Malaysia Institute of Planners (2017)

Table 1 Comparison of Actual Data of Single Storey Terrace Houses and Apartment in Penang Metropolitan

\begin{tabular}{|c|c|c|c|c|c|c|c|c|c|c|c|c|}
\hline s. & o & 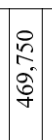 & \begin{tabular}{l|}
8 \\
8 \\
d. \\
वें
\end{tabular} & & 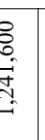 & 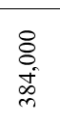 & $\left|\begin{array}{c}n \\
\stackrel{2}{2} \\
\tilde{c} \\
\tilde{c}\end{array}\right|$ & हैं & 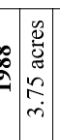 & & $\stackrel{\circ}{\circ}$ & 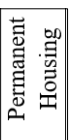 \\
\hline 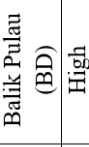 & 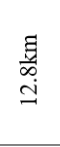 & $\mid \begin{array}{l}8 \\
0 \\
0 \\
0 \\
0 \\
\\
\end{array}$ & $\begin{array}{l}8 \\
8 \\
0 \\
0 \\
= \\
= \\
=\end{array}$ & & 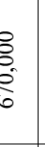 & 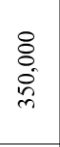 & $\begin{array}{c}8 \\
\vdots \\
\vdots \\
\text { f. }\end{array}$ & 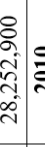 & 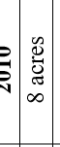 & & 8 & 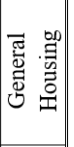 \\
\hline & & $\mid \begin{array}{l}0 \\
0 \\
0 \\
0 \\
0.0 \\
0 .\end{array}$ & 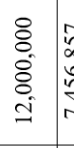 & & 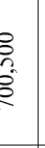 & $\begin{array}{l}8 \\
8 \\
0 \\
0 \\
0\end{array}$ & $\begin{array}{l}8 \\
\dot{0} \\
\vec{a}\end{array}$ & 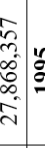 & 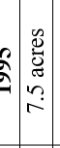 & 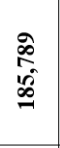 & $\stackrel{2}{3}$ & 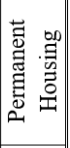 \\
\hline 营 & $\begin{array}{l}\underset{\mathrm{G}}{\mathrm{a}} \\
\stackrel{\infty}{\leftrightarrows}\end{array}$ & 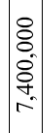 & 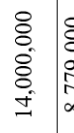 & & 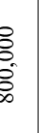 & $\begin{array}{l}8 \\
\substack{n \\
\infty \\
\infty \\
\infty}\end{array}$ & $\begin{array}{l}0 \\
0 \\
\infty \\
\infty \\
\infty \\
\infty\end{array}$ & 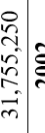 & 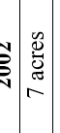 & 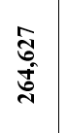 & $\nexists$ & 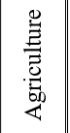 \\
\hline & 咅 & 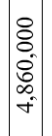 & $\begin{array}{l}8 \\
0 \\
0 \\
0 \\
0\end{array}$ & & 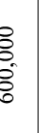 & $\begin{array}{l}\text { \& } \\
\text { oे }\end{array}$ & $\left|\begin{array}{l}n \\
a \\
i \\
n \\
m\end{array}\right|$ & 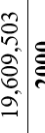 & $=$ & 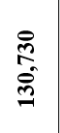 & 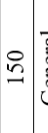 & 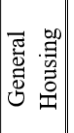 \\
\hline & 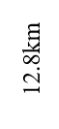 & 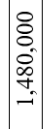 & $\begin{array}{l}8 \\
0 \\
0 \\
\overline{0}\end{array}$ & & ר. & 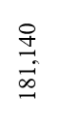 & $\begin{array}{l}8 \\
8 \\
i \\
\text { i }\end{array} \mid$ & 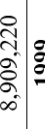 & 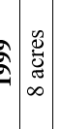 & $\begin{array}{l}\text { 亏े } \\
\text { ठิे }\end{array}$ & 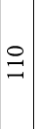 & 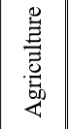 \\
\hline 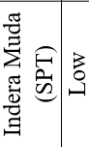 & $\frac{a}{a}$ & 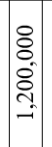 & $\begin{array}{l}8 \\
\dot{0} \\
\dot{0} \\
\vdots \\
\vdots\end{array}$ & & : & $\begin{array}{l}8 \\
8 \\
0 \\
\text { añ }\end{array}$ & $\begin{array}{l}8 \\
\vdots \\
0 \\
0 \\
m\end{array}$ & 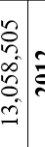 & 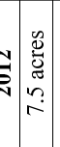 & 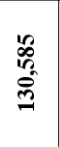 & 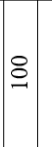 & 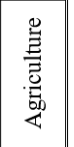 \\
\hline & 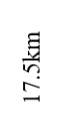 & & 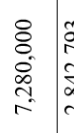 & & 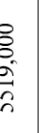 & 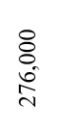 & $\left|\begin{array}{c}8 \\
\dot{0} \\
i \\
\tilde{m}\end{array}\right|$ & & 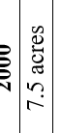 & 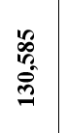 & 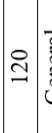 & 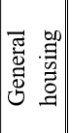 \\
\hline & 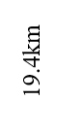 & $\begin{array}{c}0 \\
0 \\
0 \\
0 \\
0 \\
-1 \\
ت\end{array}$ & 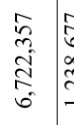 & & हू. & $\begin{array}{l}\stackrel{0}{0} \\
\stackrel{0}{\infty} \\
\stackrel{\infty}{=}\end{array}$ & 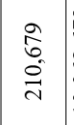 & ר" & 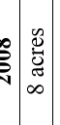 & 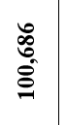 & 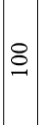 & 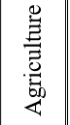 \\
\hline 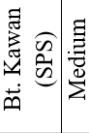 & $\begin{array}{l}\stackrel{a}{i} \\
\vec{i}\end{array}$ & 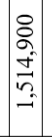 & 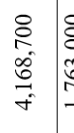 & & 炁 & 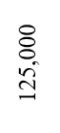 & 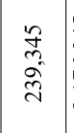 & 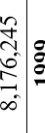 & 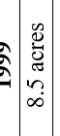 & $\begin{array}{l}\stackrel{5}{\infty} \\
\frac{1}{\infty}\end{array}$ & 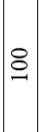 & 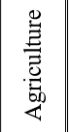 \\
\hline z & 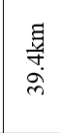 & 童 & 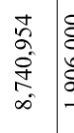 & 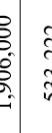 & है & 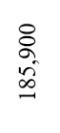 & $\begin{array}{l}8 \\
\text { o } \\
\text { is } \\
\text { 年 }\end{array}$ & 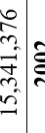 & 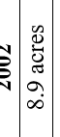 & 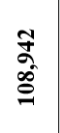 & 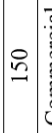 & 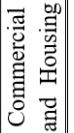 \\
\hline 类 & 0 & & & & & 4 & 品 & & & & & 寻 \\
\hline
\end{tabular}




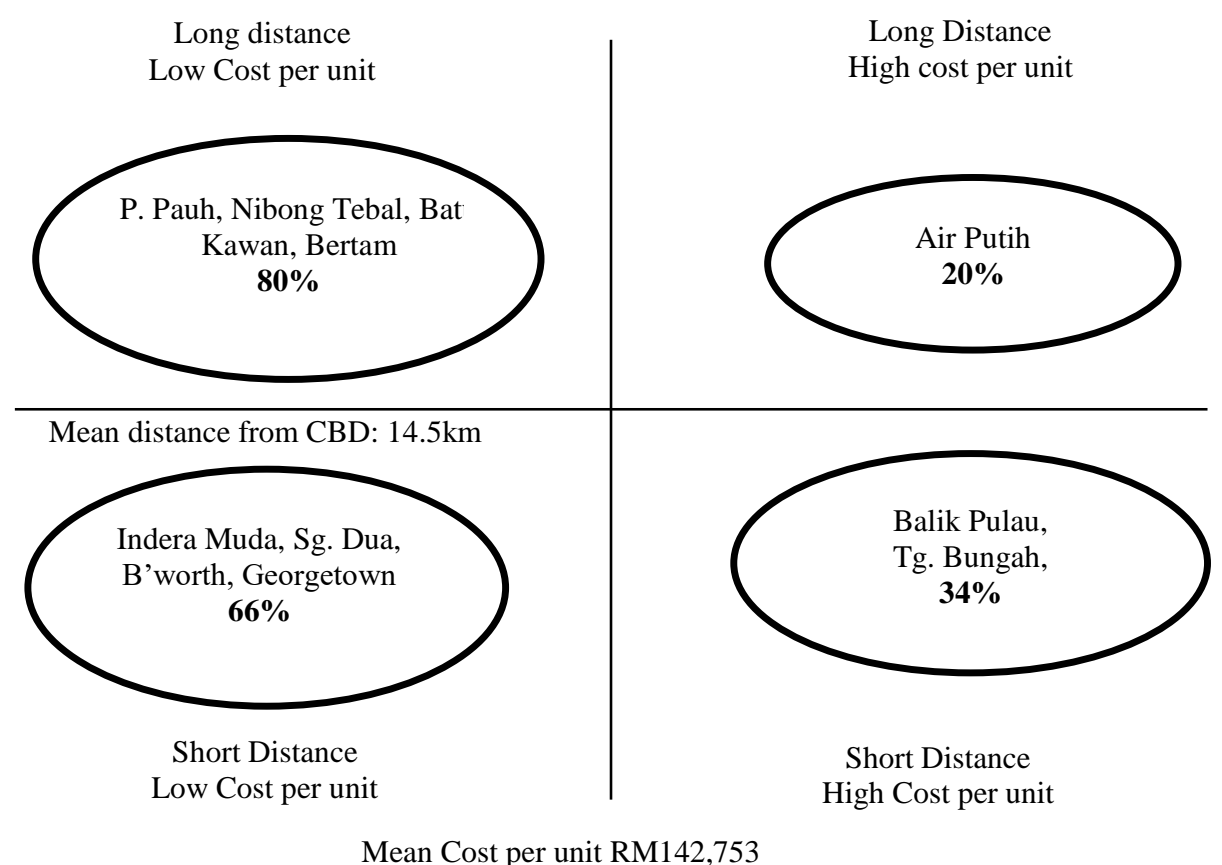

Figure 2 Cross Tabulation between Cost per Unit and Distance for 11 Housing Projects Using Normal Variables Costs

It was found that of the 5 housing projects which were located far from the CBDs, 80 percent (4 housing projects) have low cost per unit, denoting high correlation between the two variables. For the six housing projects which were near the CBDs (below the mean of 14.5 kilometres), 4 projects (66 percent) have low cost per house unit, while the other 2 have high cost per house unit, denoting weak correlation. Thus, the data show that far away housing areas have much greater proportion of lower cost housing unit, reflecting the general situation of housing prices in Malaysian urban areas.

The research then calculates additional development costs which were extension of infrastructure to the project sites as shown in Table 2. These costs included roadwork, sewerage and water supply costs calculated from each substation on the mainland and on the island. These additional costs were then added to the costs of normal variables calculated earlier. With these additional infrastructure costs, the mean price of the houses increased to RM 300,071. 
PLANNING MALAYSIA

Journal of the Malaysia Institute of Planners (2017)

Table 2 Comparison of Additional Development Cost for Single storey terrace houses

\begin{tabular}{|c|c|c|c|c|c|c|c|c|c|c|c|c|c|c|c|}
\hline $\begin{array}{ll}\stackrel{0}{0} & \widehat{P} \\
0 & 0 \\
0 & 0\end{array}$ & 咅 & 0 & $\mid \begin{array}{l}0 \\
n \\
\hat{a} \\
\dot{b} \\
+\end{array}$ & $\begin{array}{l}8 \\
8 \\
\circ \\
\circ \\
\circ \\
\dot{d}\end{array}$ & 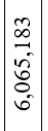 & 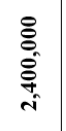 & 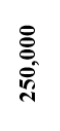 & 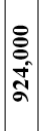 & 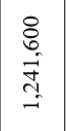 & $\begin{array}{l}8 \\
\& \\
\dot{+} \\
\infty \\
m\end{array}$ & $\begin{array}{l}m \\
\stackrel{n}{n} \\
\hat{n} \\
\tilde{n}\end{array}$ & 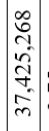 & 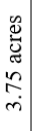 & $\begin{array}{l}\hat{\mathrm{O}} \\
\stackrel{5}{\mathrm{~F}}\end{array}$ & 宓 \\
\hline 善 $\widehat{\hat{n}}$ & : & $\begin{array}{l}\text { 睴 } \\
\text { తi }\end{array}$ & $\begin{array}{l}0 \\
\vdots \\
2 \\
0 \\
0 \\
0 \\
\end{array}$ & $\begin{array}{l}8 \\
8 \\
8 \\
\vdots \\
= \\
=\end{array}$ & 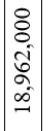 & 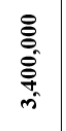 & 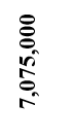 & 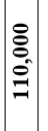 & $\begin{array}{l}8 \\
8 \\
8 \\
6\end{array}$ & $\begin{array}{l}8 \\
\vdots \\
0 \\
0 \\
m\end{array}$ & $\begin{array}{l}8 \\
\vdots \\
i n \\
\text { in }\end{array}$ & 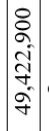 & 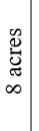 & $\begin{array}{l}\stackrel{9}{2} \\
\frac{9}{5}\end{array}$ & ঃ \\
\hline 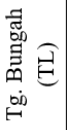 & 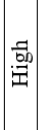 & 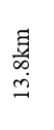 & 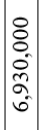 & $\begin{array}{l}8 \\
8 \\
8 \\
8 \\
i \\
i\end{array}$ & $\begin{array}{c}\hat{n} \\
\infty \\
0 \\
0 \\
0 \\
\\
-\end{array}$ & 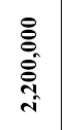 & 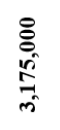 & $\begin{array}{l}0 \\
0 \\
0 \\
\infty \\
\infty \\
\infty \\
0\end{array}$ & 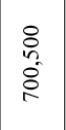 & $\begin{array}{l}\stackrel{\vdots}{0} \\
\dot{\infty} \\
\infty \\
\infty\end{array}$ & $\begin{array}{l}\stackrel{8}{0} \\
\dot{0} \\
\dot{+}\end{array}$ & $\mid \begin{array}{c}\hat{n} \\
\tilde{m} \\
\tilde{p} \\
m \\
\hat{q} \\
\hat{q}\end{array}$ & 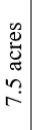 & 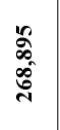 & : \\
\hline 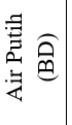 & 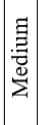 & $\begin{array}{l}\stackrel{\Xi}{\mathrm{I}} \\
\stackrel{\text { D }}{\leftrightarrows}\end{array}$ & 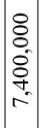 & $\begin{array}{l}8 \\
8 \\
8 \\
8 \\
\dot{0}\end{array}$ & 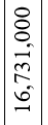 & 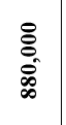 & 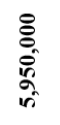 & 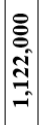 & $\begin{array}{l}8 \\
8 \\
8 \\
\infty\end{array}$ & 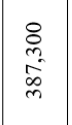 & 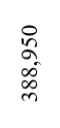 & $\mid$ & 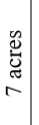 & 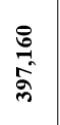 & ఫิ ఫ్ \\
\hline 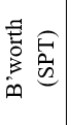 & 咅 & 0 & $\begin{array}{l}0 \\
\vdots \\
0 \\
0 \\
0 \\
\infty \\
+ \\
+\end{array}$ & $\begin{array}{l}8 \\
8 \\
8 \\
8 \\
\circ\end{array}$ & 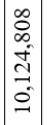 & 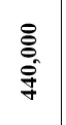 & 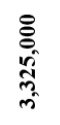 & 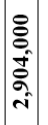 & $\begin{array}{l}8 \\
: \\
8 \\
8\end{array}$ & $\begin{array}{l}\text { \&े } \\
\text { ôे } \\
\text { ñ. }\end{array}$ & 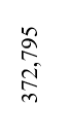 & $\begin{array}{c}0 \\
0 \\
0 \\
0 \\
0 \\
0 \\
0 \\
n \\
0\end{array}$ & 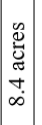 & $\begin{array}{l}\bar{a} \\
\hat{\tilde{n}}\end{array}$ & in \\
\hline 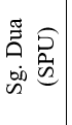 & 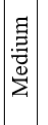 & 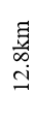 & 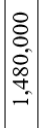 & $\begin{array}{c}8 \\
8 \\
8 \\
\dot{0} \\
\text { in }\end{array}$ & 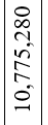 & 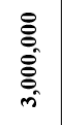 & 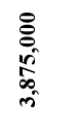 & 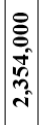 & 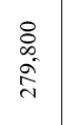 & 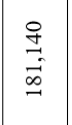 & $\begin{array}{l}\text { \& } \\
\text { î̀ } \\
\text { i }\end{array}$ & 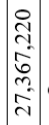 & $\mid \begin{array}{l} \\
\mathscr{w} \\
0 \\
\tilde{c} \\
\infty \\
\infty\end{array}$ & 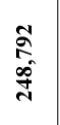 & 이 \\
\hline 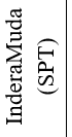 & $\mid \begin{array}{l}0 \\
0 \\
\vdots \\
\ddots\end{array}$ & $\begin{array}{l}\text { 貝 } \\
\dot{m}\end{array}$ & 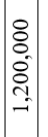 & $\begin{array}{l}8 \\
8 \\
0 \\
8 \\
6 \\
-1\end{array}$ & $\begin{array}{l}0 \\
0 \\
n \\
\infty \\
œ \\
\infty \\
\infty \\
0\end{array}$ & $\begin{array}{l}\text { : } \\
\text { : } \\
\text { : }\end{array}$ & 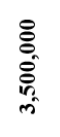 & 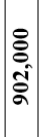 & ¿ & $\begin{array}{l}8 \\
\text { in }\end{array}$ & $\begin{array}{l}\text { ठे } \\
\text { ले } \\
\text { ले }\end{array}$ & 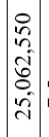 & 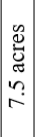 & 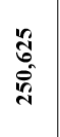 & $8 \cong$ \\
\hline 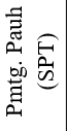 & 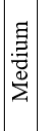 & $\begin{array}{l}\frac{\mathrm{G}}{2} \\
\stackrel{2}{=}\end{array}$ & $\mid \begin{array}{c}0 \\
2 \\
0 \\
0 \\
0 \\
0 \\
-1\end{array}$ & $\begin{array}{l}8 \\
8 \\
\circ \\
\stackrel{0}{1} \\
\stackrel{\sim}{\sim}\end{array}$ & $\begin{array}{l}0 \\
2 \\
0 \\
0 \\
0 \\
0 \\
-\end{array}$ & 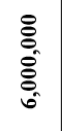 & 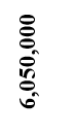 & 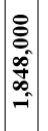 & $\begin{array}{l}8 \\
\stackrel{\circ}{\circ} \\
\text { in }\end{array}$ & 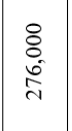 & $\begin{array}{l}\stackrel{8}{0} \\
\text { î } \\
m\end{array}$ & 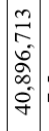 & 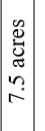 & $\begin{array}{l}0 \\
0 \\
0 \\
O \\
\stackrel{5}{m}\end{array}$ & $£$ \\
\hline 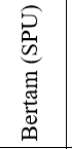 & 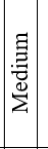 & $\begin{array}{l}\text { 冒 } \\
\stackrel{-}{-}\end{array}$ & $\mid$\begin{tabular}{l}
0 \\
0 \\
$m$ \\
0 \\
$\infty$ \\
\hdashline \\
$\exists$
\end{tabular} & $\begin{array}{l}\sqrt[n]{n} \\
0 \\
i \\
i \\
\hat{i}\end{array}$ & 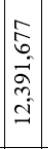 & 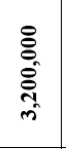 & 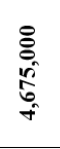 & 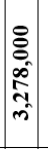 & $\begin{array}{l}\text { ¿े } \\
\text { ते } \\
\text { हn }\end{array}$ & 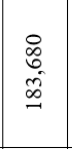 & $\begin{array}{l}\hat{\widehat{\hat{\sigma}}} \\
\text { } \\
\text { ते }\end{array}$ & 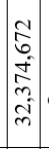 & 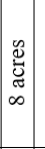 & 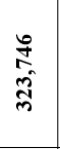 & ¿ \\
\hline 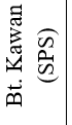 & \begin{tabular}{|c|} 
\\
0 \\
0 \\
\end{tabular} & $\frac{\text { 塄 }}{\stackrel{\sim}{N}}$ & $\begin{array}{l}8 \\
\swarrow \\
\dot{q} \\
\\
-\end{array}$ & $\begin{array}{l}8 \\
\stackrel{0}{0} \\
o \\
0 \\
+ \\
+\end{array}$ & 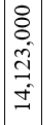 & 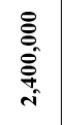 & 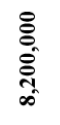 & 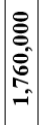 & $\begin{array}{l}8 \\
0 \\
i \\
0 \\
0 \\
\text { n. }\end{array}$ & $\begin{array}{l}8 \\
\vdots \\
\\
\text { İ }\end{array}$ & $\begin{array}{l}\text { 告 } \\
\text { mे } \\
\text { ले }\end{array}$ & 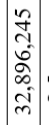 & $\mid$ & 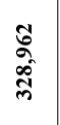 & ‡ \\
\hline 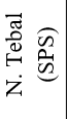 & 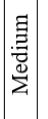 & $\begin{array}{l}\text { 貝 } \\
\text { 品 }\end{array}$ & $\mid \begin{array}{l}8 \\
\vdots \\
0 \\
0 \\
0 \\
m \\
m\end{array}$ & $\begin{array}{l}\stackrel{+}{2} \\
2 \\
o \\
\stackrel{+}{+} \\
\infty\end{array}$ & 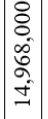 & 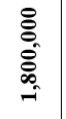 & 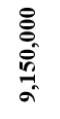 & 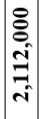 & $\begin{array}{l}\text { ה̃ } \\
\text { } \\
\tilde{n}\end{array}$ & $\begin{array}{l}8 \\
\vdots \\
\text { nो } \\
\infty \\
=\end{array}$ & $\begin{array}{l}8 \\
\text { on } \\
\text { nn } \\
\text { and } \\
\text { nd }\end{array}$ & $\begin{array}{c}1 \\
0 \\
0 \\
0 \\
6 \\
b \\
7 \\
7\end{array}$ & 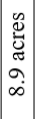 & 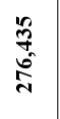 & 0 \\
\hline 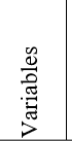 & 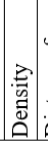 & 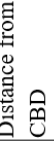 & 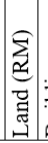 & 昜 & 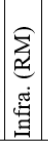 & 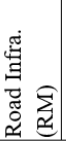 & 总 & 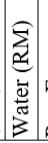 & 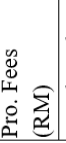 & 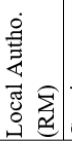 & 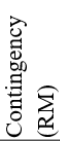 & $\mid$ & & 吾 & $\mid \begin{array}{l}5 \\
\dot{0} \\
\dot{z} \\
z\end{array}$ \\
\hline
\end{tabular}


Shahriza Osman, Jamalunlaili Abdullah, \& Abdul Hadi Nawawi

The Financial Costs of Urban Sprawl: Case Study of Penang State

The addition of infrastructure costs to the "normal" costs has produced different rates of cost increase for different regions. Those on the island demonstrated an increase between 30 to 42 percent in total costs per house unit. On the mainland, the rate of increase was much higher. For instance, the increase in Seberang Perai Utara was about 68 percent, while the increase in cost per unit in Seberang Perai Selatan was between 60 to 75 percent. This validates Torren's (2006) assertion that farther away development actually increases the cost of housing unit due to additional cost of providing infrastructure.

The new costs were correlated with the distance and are shown in Figure 3. Interestingly, the results for long distance housing projects are opposite of those shown in Figure 2.

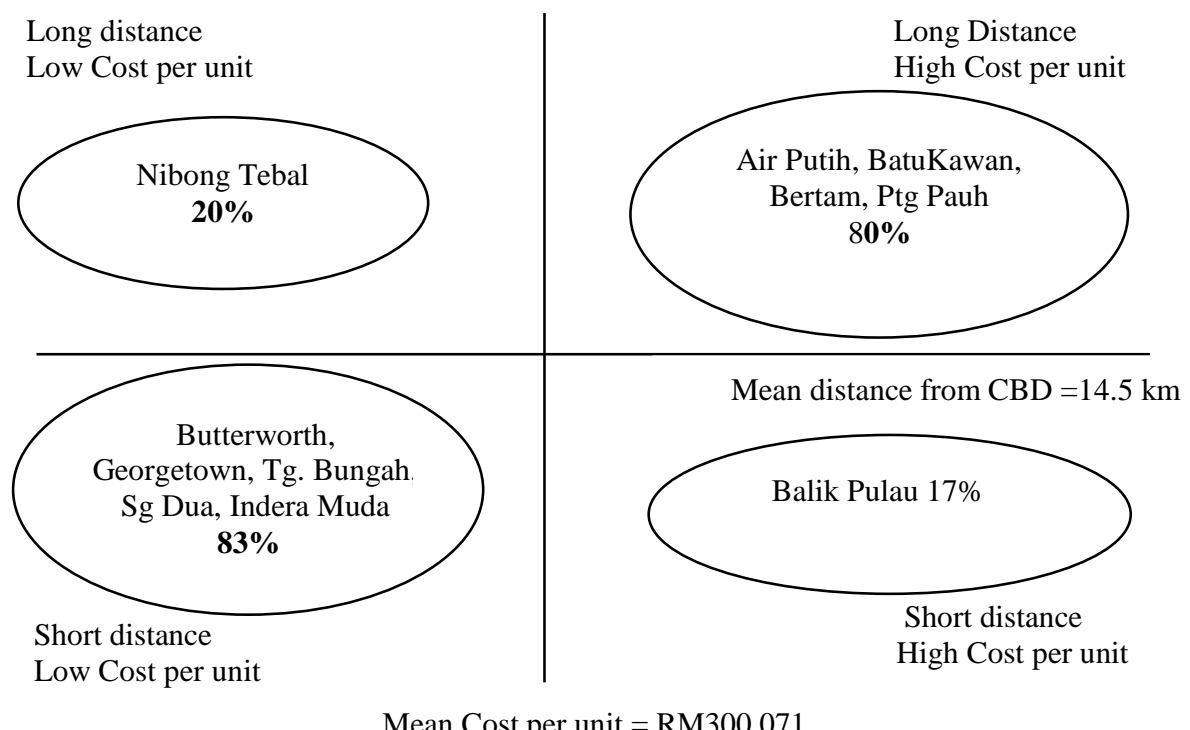

Figure 3 Cross Tabulation between Distance and Cost per Unit for 11 Housing Projects after Adding the Costs of Extending Infrastructure to the Sites.

For far away (long distance development), after adding the cost of extending infrastructure to the housing project, it was found that 80 percent (4 out of 5 projects) have now become high cost housing per unit. In Figure 2, without adding the infrastructure extension cost, only 20 percent ( 1 out of 5 projects) were high cost. More significantly, for the short distance development, after adding the infrastructure extension cost, 83 percent of the housing project were low cost per unit housing projects. These data provide strong correlation between distance and cost of housing per unit. After adding the cost of extending 
PLANNING MALAYSIA

Journal of the Malaysia Institute of Planners (2017)

infrastructure to the housing projects, the developments closer to CBDs have lower cost per unit compared to housing developments far away from the CBDs.

\section{CONCLUSION}

The paper provides empirical proof that development away from city centres are actually more costly compared to those near the city centres when the cost of extending infrastructure are included in the total costs calculation. Therefore, this study proposes that the additional cost of infrastructure such as road and sewage need to be accounted for. These are the infrastructure costs which, at the moment, are not included in the calculation of the Gross Development Value (GDV) of a development project. The addition of infrastructure extension cost is to show the true cost of developing a housing project.

The findings of this study support contention by Burchell (1990) and other scholars that sprawl actually costs more than compact development near the city centres. The costs of extending infrastructure tend to be higher than the additional costs of land in or near the city. However, since these infrastructure extensions costs are it being paid for by the state, they are not included in the calculation of development costs by the private developers. Hence, many people have the impression that developing projects such as housing areas away from the city centres entail lower costs. The reality is that cost of sprawl studies show that substantial infrastructure costs savings can be achieved by increasing urban densities and locating new development near existing built-up areas.

Similar study can be extended to other housing projects in Penang State in order to arrive at comprehensive findings on the cost of sprawl. Similar study in the Klang valley and Johor Bharu would also enrich the understanding about the cost of urban sprawl in Malaysia.

\section{REFERENCES}

Ahris Yaakup, \& Susilawati Sulaiman (2005). GIS as new approach and method in preparing and implementing the development plan in Malaysian planning system. $\quad$ Retrieved from http://eprints.utm.my/622/2/GIS_As_New_Approach_And_Method\%28_\%29 Ahris_Yaakup.pdf

Abdullah, J. (2003). The suburbanisation of the Kuala Lumpur Metropolitan Region. Planning Malaysia, 1, 119-126.

Abdullah, J. (2009, November). Rekabentuk bandar kompak ke arah pembangunan mapan. Seminar Kebangsaan Rupabandar Malaysia - Mengekal Warisan Membina Identiti. November 28-29, 2007, Lumut, Perak.

Abdullah, J., Yahaya, M. Z., Yunus M. Z, \& Safudin, M. S. (2009). Urban sprawl in Malaysia: evidences from three largest metropolitan areas. Planning Malaysia, 7, 69-82.

Bryant, L., \& Eves, C. (2014). The link between infrastructure charges and housing affordability in Australia: where is the empirical evidence? Australian Planner, 51(4), 307-317. 
Shahriza Osman, Jamalunlaili Abdullah, \& Abdul Hadi Nawawi

The Financial Costs of Urban Sprawl: Case Study of Penang State

Burchell, Robert W. (1990). Fiscal impact analysis: state of the art and state of the practice. In S. G. Robinson (Ed.), Financing growth: who benefits? Who pays? And how much? Chicago: Government Finance Officers Association

Burchell, R. W., \& Shad, N. A. (1998). Evolution of the sprawl debate in the United States. Hastings West-Northwest Journal of Environmental Law \& Policy, 5 , 137.

Duncan, J. G. (1992). Preserving rural landscape in the midst of suburban sprawl (Masters Thesis). University of Rhode Island.

Ewing, R., Pendall, R., \& Chen, D. (2002). Measuring urban sprawl and its impacts.

Frank, J. E. (1989). The cost of alternative development pattern: a review of the literature. Washington, DC: Urban Land Institute.

Isard, W., \& Coughlin, R. E. (1957). Municipal cost and revenues resulting from growth. Wellesley, Massachusetts: Chandler-Davis.

Ladd, H. (1998). Local government tax and land use policies in the United States: understanding the links. In W. Oates (Ed.), Studies in fiscal federalism and state location. Cambridge, MA: Lincoln institute of Land policy.

Noor, N. M., Asmawi, M. Z., \& Rusni, N. A. (2014). Measuring urban sprawl on geospatial indices characterized by leap frog development using remote sensing and GIS techniques. In IOP Conference Series: Earth and Environmental Science, 18(1). IOP Publishing (Online).

Noor, N. M., \& Rosni, N. A. (2013). Determination of spatial factors in measuring urban sprawl in Kuantan using remote sensing and GIS. Procedia-Social and Behavioral Sciences, 85, 502-512.

Ojima, R. (2007). Mobility, urban sprawl and environmental risk in agglomerations. PRIPODE Workshop on Urban Population-Development-Environment Dynamics. June 11-13, 2007, Nairobi, Kenya.

RERC- Real Estate Research Corporation (1974). The costs of sprawl: detailed cost analysis. Washington D.C.: U.S. Government Printing Office.

Sabri, S., \& Yakuup, A. (2008). Multi-criteria decision making for urban sprawl, using Analytic Network Process and GIS, case of Iskandar Malaysia Region. Map Asia 2008. August 18-21, 2008, Kuala Lumpur, Malaysia.

Safudin, M. S. (2007). Urban sprawl: case study of Johor Bahru (Dissertation). Universiti Teknologi MARA, Shah Alam, Selangor.

Speir, C., \& Stephenson, K. (2002). Does sprawl cost us all? Isolating the effects of housing patterns on public water and sewer costs. Journal of the American Planning Association, 68(1), 56-70.

Thomson, G., Hoffman, J., \& Staniforth, S. (2003). Measuring the success of environmental education programs. Ottawa: Canadian Parks and Wilderness Society and Sierra Club of Canada.

Torrens, P. M. (2006). Geosimulation and its application to urban growth modeling. In J. Portugali (Ed.), Complex artificial environments (pp. 119-134). London: Springer.

Yahaya, M. Z. (2006). Urban growth and sprawl in Kuala Lumpur Metropolitan Region (Dissertation). Universiti Teknologi MARA, Shah Alam, Selangor. 